\title{
Misinformation and attitudes towards COVID-19 vaccines among Polish migrants in the UK: a clinician's perspective
}

\author{
Maciej D. Zatonski
}

Associate of the Institute - European Observatory of Health Inequalities, Calisia University, Kalisz, Poland

\begin{abstract}
Rapid vaccine-induced population immunity is the key global strategy to control COVID-19 pandemic. However, coordination of the deployment of the vaccination programmes varies significantly between countries. In the meantime, we have witnessed an unprecedented media coverage of the deployment of the COVID-19 vaccination programmes. Unfortunately, most of those reports were incomplete, poorly understood, and misguided - often fuelling growing vaccine hesitancy among the public caused by unfounded fears and confusion. For the last 10 years the author of this paper has lived and worked as a healthcare professional in the UK. In December 2019 he has volunteered to support the rollout of the mass vaccination programme against COVID-19 - the largest civilian logistical operation ever conducted in Britain. This article describes his personal observations on how Poles living in the UK approach the vaccination programme, and the challenges related to immunisation of Polish citizens in the UK, including vaccine (mis)information.
\end{abstract}

KEY WORDS: COVID-19, vaccines, vaccine deployment, vaccine acceptance, vaccines hesitancy.

ADDRESS FOR CORRESPONDENCE: Maciej Dariusz Zatonski, Associate of the Institute - European Observatory of Health Inequalities, Calisia University, 4 Nowy Świat Str., 62-800 Kalisz, Poland, e-mail: public.mz@gmail.com

From the earliest stages of the COVID-19 pandemic, the development of safe and effective vaccines against severe acute respiratory syndrome coronavirus-2 (SARSCoV-2), the viral cause of COVID-19, has been an essential component of any strategy to control the virus, the disease, and its effects. Rapid vaccine-induced population immunity is therefore the key global strategy to control COVID-19 pandemic. Vaccination programmes must maximise early impact, particularly with accelerated spread of new variants [1]. The United Kingdom was amongst the countries hit the hardest during the first waves of the pandemic in 2020 (https://www.bbc.com/news/business-55143285).

Coordination of the deployment of the vaccination programmes however varies significantly between countries. Current evidence suggests that in a public health emergency vaccine deployment should be prioritised over the efficacy of protection in single individuals, especially during periods of potential shortages of available vaccines [2]. Such approach was introduced in the United Kingdom, where the efforts were focused on administering at least one dose of the vaccine to as large number of priority individuals as possible. The administration of the second dose was delayed until more stock became available and until the country's capability to vaccinate large numbers of people in a short amount of time gradually increased. Other countries (such as Poland) have opted for maximising individual protection (i.e. prioritising the administration of both doses of the vaccine), while leaving a significantly large fraction of the population entirely unvaccinated. Countries with smaller general population (like Israel) could afford to maximise both.

In the meantime, we have witnessed an unprecedented media coverage of the deployment of the COVID-19 vaccination programme. Daily news portals were filled with information about vaccine trials, vaccine safety assessments, or differences in how various countries 
approached the public health crisis. Unfortunately, most of those reports were incomplete, poorly understood and misguided - often fuelling growing vaccine hesitancy among general public caused by unfounded fears and confusion. For example, a decision made by numerous EU member states to suspend the use of the vector ChAdOx1 nCoV-19 vaccine (Oxford/AstraZeneca vaccine) following concerns it could cause extremely rare thromboembolic events, has dramatically damaged the already poor perception of the safety of COVID-19 vaccines and substantially contributed to the pre-existing hesitancy. In the weeks leading up to suspension of viral vector vaccines, the weekly increase in vaccine uptake in the EU was close to $2 \%$ of the adult population. Since the suspended vaccine represents $25 \%$ of doses used in the $\mathrm{EU}$; therefore, the suspension could have potentially prevented up to 2 million vulnerable people from receiving a preventive COVID-19 vaccine [3]. A delay of one week during deployment of vaccination with a COVID-19 vaccine, using the most conservative estimates, could have translated to direct failure to prevent up to 3000 COVID-19 cases, 50 to 80 COVID-19 related deaths, and cause at least 30 COVID-19 related thromboembolic events.

Public reporting on vaccines is not the only factor contributing to large differences in how countries approach and succeed in their attempts to tackle the ongoing pandemic. For the last 10 years I have lived and worked as a healthcare professional in the UK. In December 2019 I have volunteered, together with my wife and hundreds of other doctors, nurses and pharmacists, to support the rollout of the mass vaccination programme against COVID-19 - the largest civilian logistical operation ever conducted in Britain. For the last 4 months, at least few times per week, after finishing my daily work, I would go to the local vaccination centre and volunteer my time and skills to help vaccinate hundreds of people every day, including weekends and public holidays.

This experience has given me the unique opportunity to observe how people respond to vaccinations. I have observed a stark contrast between how British patients approach the vaccinations in comparison to Polish patients. On average it would take much longer to vaccinate a Polish person in the UK. Most of my compatriots would arrive to their appointments visibly confused and hesitant. They often appeared scared or very concerned and were asking multiple questions. Few of the enquires however seemed related to what is relevant during the vaccination. Polish citizens patients appeared to be primarily concerned by made-up or completely unfounded fears such as:

- "I don't want to be vaccinated, because I will go to hell, as vaccines are made from aborted foetuses",

- "I don't need to vaccinate, because UK has already achieved herd immunity",

- "If I will not be able to walk without a mask, I don't want nor need a vaccine",
- "I am afraid that my DNA will be mutated, and I will die or get cancer",

- "I don't trust the clinical trials and regulators; therefore, I don't trust in the vaccine",

- "I am worried that the vaccine will make me infertile".

Up to date, I have not received a single similar question from a British person in my vaccination clinic. It is difficult to imagine that such absurd worries and concerns would be up by the individuals themselves, and their volume and repetitive nature suggests that many of my Polish patients have been exposed to similar types of misinformation from particular sources. Many Poles in the UK do not actively participate in public life in the UK and rarely consume official news sources. Hardly any official health information is available in the UK in Polish language or is culturally tailored for the needs of Polish patients. It should be noted that the questions listed above came from people who have decided to either register for or accept a vaccination appointment. There are many others who have never showed up in the first place. Estimates suggest that in many areas a significant proportion of Polish nationals remain unvaccinated, while in respective age groups over $99 \%$ of Britons have received their COVID-19 vaccines [4].

In my local vaccination centre, $99.5 \%$ of people over the age of 75 have received their vaccine, and more than $90 \%$ of those aged between 50-75. Among the unvaccinated ones, a significant portion are people with Polish-sounding names. The problem must be wider, as in late February I was approached by Public Health England and the UK Cabinet Office to support education of Polish citizens in the UK on vaccines and the pandemic. For the last 2 months I have been volunteering my time as an expert during media events, radio interviews, Q\&A sessions with the Polish community, social media interviews, and other events sponsored by the UK government and aimed at reaching Polish citizens with reliable information about the COVID-19 vaccines.

This problem is not isolated to Polish people living in the UK, as similar patterns of vaccine misinformation are observed among other minorities as well, and those attitudes are not only present in the UK. It seems that vaccine acceptance among Polish nationals living in Poland is also relatively low, with up to $30 \%$ of Poles declaring that they will refuse to receive a COVID-19 vaccine [5].

In the UK, the government and the public healthcare workers consistently sent reliable, factual, and accurate messages to the public regarding vaccines, vaccine safety and the progress of vaccine deployment. This messaging is also aligned with the planned relaxation of travel restrictions and business closures. British politicians and even members of the Royal Family patiently waited for their turn to be vaccinated. Elderly people, frontline healthcare workers and residents of care homes started to receive their vaccines before the politicians, ministers, 
or even the Queen. The priority was assigned based on scientifically calculated levels of risk, and there was no "skipping the queue" that we have witnessed in Poland. In fact, any attempt to do so were regarded as selfish and unethical among the British public.

Polish patients no longer have to die and suffer consequences from COVID-19. The successful saving of Polish lives might depend largely on the acceptance of the available vaccines.

\section{DISCLOSURE}

The author reports no conflict of interest.

\section{References}

1. Joint Committee on Vaccination and Immunisation. Optimising the COVID-19 vaccination programme for maximum shortterm impact. Jan 26, 2021. Available from: https://www.gov.uk/ government/publications/prioritising-the- first-covid-19-vaccinedose-jcvi-statement/ optimising-the-covid-19-vaccination- programme-for-maximum-short-term-impact (accessed: 21 May 2021).

2. Palitel DA, Schwartz J, Zheng A, Walensky RP. Clinical outcomes of a COVID-19 vaccine: implementation over efficacy. Health Aff (Millwood) 2021; 40(1): 42-52.

3. European Centre for Disease Prevention and Control. COVID-19 situation update for the EU/EEA, as of 26 March 2021. Mar 26, 2021. Available from: https://www.ecdc.europa.eu/en/cases-2019ncov-eueea (accessed: 21 May 2021).

4. National Health Service. COVID-19 Vaccinations. May 20, 2001. Available from: https://www.england.nhs.uk/statistics/statistical-work-areas/covid-19-vaccinations/ (accessed: 21 May 2021)

5. Warsaw's Institute for Social Research and Market (IBRIS). Available from: https://wiadomosci.radiozet.pl/Koronawirus/Szczepienia-na-COVID-19.-Sondaz-IBRIS.-Polacy-obawiaja-sie-powiklan (accessed: 16 May 2021) 